\title{
Preparation and Characterization of Deep Eutectic Solvents That Can Be Used in $\mathrm{CO}_{2}$ Absorption Processes ${ }^{+}$
}

\author{
Eliza-Gabriela Mihăilă 1,2, Cristian Florian Dincă 2,*, Marian Deaconu 1, Dana Simona Vărășteanu 1, \\ Irina Elena Chican ${ }^{1}$, Daniel Preda ${ }^{1}$, Diana Constantinescu-Aruxandei 1,* and Florin Oancea 1,* \\ 1 National Institute for Research \& Development in Chemistry and Petrochemistry-ICECHIM, \\ 060021 Bucharest, Romania; mihaila.eliza.gabriela@gmail.com (E.-G.M.); deaconu319@yahoo.com (M.D.); \\ dana.varasteanu@icechim.ro (D.S.V.); irina-elena.chican@icechim.ro (I.E.C.); daniel.preda@icechim.ro (D.P.) \\ 2 Power Engineering Faculty, University Politehnica Bucharest, 060042 Bucharest, Romania \\ * Correspondence: cristian.dinca@upb.ro (C.F.D.); Diana.C.Aruxandei@gmail.com (D.C.-A.); \\ florin.oancea@icechim.ro (F.O.) \\ + Presented at the 16th International Symposium "Priorities of Chemistry for a Sustainable Development" \\ PRIOCHEM, Bucharest, Romania, 28-30 October 2020.
}

Published: 10 November 2020

Keywords: green solvents; deep eutectic solvents; characterization; $\mathrm{CO}_{2}$ absorption

$\mathrm{CO}_{2}$ emissions are well-known for creating a lot of environmental issues, at a global scale. One of the most-used technologies for $\mathrm{CO}_{2}$ capture is the post-combustion method, which consists of separating the $\mathrm{CO}_{2}$ from the combustion gases, using a solvent, usually amine. In this study, we propose alternative solvents: DESs (deep eutectic solvents) [1], based on choline chloride and amine, and switchable hydrophilicity DESs, based on fatty acids and diluted amine solutions. For standard DESs, choline chloride ( $\mathrm{ChCl})$ was used as hydrogen bond donor (HBD), and amines (monoethanolamine (MEA), diethanolamine (DEA), and triethanolamine (TEA)) as hydrogen bond acceptors (HBAs) [2,3], in different molar ratios: 1:5, 1:6, 1:8, and 1:10. All the components were precisely weighed and mixed at $300 \mathrm{RPM}, 60^{\circ} \mathrm{C}$, until a clear solution was obtained. For switchable hydrophilicity DESs, the hydrophobic part is represented by a hydrophobic DES [4], made up of octanoic acid (OA) and other three acids, successively: dodecanoic (DA), myristic (MA), and stearic acid (SA), each in a 3:1 molar ratio. The hydrophilic phase consists of $10 \%$ amine solution [5] MEA, DEA, and TEA. The two phases were blended at 1:13 v/v oil-water, and then analyzed. The obtained solvents were in liquid state at room temperature, except for the OA-SA DES. After adding the amine solutions, the OA-SA-amine DESs were also liquid. All DESs were characterized using $\mathrm{pH}$, density, viscosity, electrical conductivity, refraction index, surface tension, FTIR, and NMR. The prepared DESs are used for solubility tests to determine $\mathrm{CO}_{2}$ absorption capacity. The hybrid DES-amines mixtures have higher absorption capacity than the ChCl-based DESs and the conventional 30\% MEA - up to double $\mathrm{CO}_{2}$ absorption capacity. Comparing the three amine types, MEA gives the best results, even in hybrid DESs. DESs represent a new green solution for $\mathrm{CO}_{2}$ absorption. Their advantages consist of cost-effective solvent price, easy preparation, easy reuse, and regeneration. Based on the previous observations that amines and DESs can absorb $\mathrm{CO}_{2}$, we developed combinations between them that improve the $\mathrm{CO}_{2}$ absorption capacity and that can be tailored for controlled release in various applications.

Acknowledgements: The work on this paper was supported by the Government of Romania, Ministry of Education and Research, Project PFE 31/2018 - TRANS-CHEM, and Project 296PED/2020 - ASOCIAT, and Project 10PCCDI/2018-PRO-SPER funded by UEFISCDI. It was also funded by the UEFISCDI-Ministry of Education and Research, contract no. 51675/09.07.2019 POCU/380/6/13, A-Succes SMIS code 125125. 


\section{References}

1. Paiva, A.; Craveiro, R.; Aroso, I.; Martins, M.; Reis, R.L.; Duarte, A.R.C. Natural deep eutectic solvents -Solvents for the 21st century. ACS Sustain. Chem. Eng. 2014, 2, 1063-1071, doi:10.1021/sc500096j.

2. Adeyemi, I.; Abu-Zahra, M.R.M.; Alnashef, I. Novel Green Solvents for CO2 Capture. Energy Procedia 2016, 114, 2552-2560, doi:10.1016/j.egypro.2017.03.1413.

3. Adeyemi, I.; Abu-Zahra, M.R.M.; Alnashef, I. Experimental Study of the Solubility of CO2 in Novel Amine Based Deep Eutectic Solvents. Energy Procedia 2017, 105, 1394-1400, doi:10.1016/j.egypro.2017.03.519.

4. Chen, Q.; Wang, L.; Ren, G.; Liu, Q.; Xu, Z.; Sun, D. A fatty acid solvent of switchable miscibility. J. Colloid Interface Sci. 2017, 504, 645-651, doi: 10.1016/j.jcis.2017.06.011.

5. Sed, G.; Cicci, A.; Jessop, P.G.; Bravi, M. A novel switchable-hydrophilicity, natural deep eutectic solvent (NaDES)-based system for bio-safe biorefinery. RSC Adv. 2018, 8, 37092-37097, doi:10.1039/c8ra08536f.

Publisher's Note: MDPI stays neutral with regard to jurisdictional claims in published maps and institutional affiliations.

(C) 2020 by the authors. Licensee MDPI, Basel, Switzerland. This article is an open access article distributed under the terms and conditions of the Creative Commons Attribution (CC BY) license (http://creativecommons.org/licenses/by/4.0/). 